\title{
Follow-up plasma apolipoprotein E levels in the Australian Imaging, Biomarkers and Lifestyle Flagship Study of Ageing (AIBL) cohort
}

Veer B Gupta ${ }^{1,2+}$, Andrea C Wilson ${ }^{1,2+}$, Samantha Burnham ${ }^{3}$, Eugene Hone ${ }^{1,2}$, Steve Pedrini ${ }^{1,2}$, Simon M Laws ${ }^{1,2,4}$, Wei Ling Florence Lim ${ }^{1,2}$, Alan Rembach ${ }^{5}$, Stephanie Rainey-Smith ${ }^{1,2}$, David Ames, ${ }^{6,7}$, Lynne Cobiac $^{8}$, S Lance Macaulay 9 , Colin L Masters ${ }^{4,10,11}$, Christopher C Rowe ${ }^{12}$, Ashley I Bush ${ }^{4,10,11}$, Ralph N Martins 1,2,4,13* and for the AIBL Research Group

\begin{abstract}
Introduction: Alzheimer's disease (AD) is a growing socioeconomic problem worldwide. Early diagnosis and prevention of this devastating disease have become a research priority. Consequently, the identification of clinically significant and sensitive blood biomarkers for its early detection is very important. Apolipoprotein $E(A P O E)$ is a well-known and established genetic risk factor for late-onset AD; however, the impact of the protein level on AD risk is unclear. We assessed the utility of plasma ApoE protein as a potential biomarker of AD in the large, well-characterised Australian Imaging, Biomarkers and Lifestyle Study of Ageing (AIBL) cohort.

Methods: Total plasma ApoE levels were measured at 18-month follow-up using a commercial bead-based enzyme-linked immunosorbent assay: the Luminex XMAP human apolipoprotein kit. ApoE levels were then analysed between clinical classifications (healthy controls, mild cognitive impairment (MCI) and $A D$ ) and correlated with the data available from the AIBL cohort, including but not limited to APOE genotype and cerebral amyloid burden.

Results: A significant decrease in ApoE levels was found in the AD group compared with the healthy controls. These results validate previously published ApoE protein levels at baseline obtained using different methodology. ApoE protein levels were also significantly affected, depending on APOE genotypes, with $\varepsilon 2 / \varepsilon 2$ having the highest protein levels and $\varepsilon 4 / \varepsilon 4$ having the lowest. Plasma ApoE levels were significantly negatively correlated with cerebral amyloid burden as measured by neuroimaging.
\end{abstract}

Conclusions: ApoE is decreased in individuals with AD compared with healthy controls at 18-month follow-up, and this trend is consistent with our results published at baseline. The influence of APOE genotype and sex on the protein levels are also explored. It is clear that ApoE is a strong player in the aetiology of this disease at both the protein and genetic levels.

\footnotetext{
*Correspondence: r.martins@ecu.edu.au

${ }^{\dagger}$ Equal contributors

${ }^{1}$ Centre of Excellence in Alzheimer's Disease Research and Care, School of Medical Sciences, Edith Cowan University, 270 Joondalup Drive, Joondalup 6027, Australia

${ }^{2}$ McCusker Alzheimer's Research Foundation, Hollywood Medical Centre, 85

Monash Avenue, Suite 22, Nedlands 6009, Australia

Full list of author information is available at the end of the article
} 


\section{Introduction}

Current research in the area of Alzheimer's disease (AD) indicates an urgent need for the discovery and validation of sensitive and specific protein biomarkers for the early detection and treatment of this devastating disease [1,2]. Pathological changes in brain amyloid- $\beta$ (A $\beta$ ) protein deposits visualised by neuroimaging are captured up to 15 years prior to the manifestation of clinical symptoms [3-5]. With definitive diagnosis possible only post-mortem, it is critical that more inexpensive and widely accessible strategies be developed to capture these changes early. Blood-based biomarkers are currently being mined for utility in detecting preclinical AD, where the goal is to develop a screening tool in the form of a routine blood test for early diagnosis.

Apolipoprotein E (ApoE) is a well-defined genetic risk factor for late-onset $\mathrm{AD}$ [6]. The human $A P O E$ gene has three polymorphic alleles-namely $\varepsilon 2, \varepsilon 3$ and $\varepsilon 4$ [7]-where an individual acquires two alleles resulting in six different phenotypes: $\varepsilon 2 / \varepsilon 2, \varepsilon 2 / \varepsilon 3, \varepsilon 3 / \varepsilon 3, \varepsilon 2 /$ $\varepsilon 4, \varepsilon 3 / \varepsilon 4$ and $\varepsilon 4 / \varepsilon 4$. Importantly, approximately $50 \%$ of AD patients carry the $\varepsilon 4$ allele (compared with $14 \%$ in the general population), with the majority being heterozygotes $(\varepsilon 3 / \varepsilon 4[8,9])$. The APOE- $\varepsilon 4$ allele has been implicated in many AD pathological pathways. Furthermore, the number of inherited $\varepsilon 4$ alleles is associated with both increased disease risk and decreased average age of onset compared with inheritance of the $\varepsilon 2$ or $\varepsilon 3$ alleles [8].

Biologically, the ApoE protein is known to influence lipid homeostasis by regulating lipid transport, such as cholesterol, in an isoform-dependent manner [10-12]. The differences between the three ApoE isoforms are based on two amino acids that affect its structure and hence the interaction and binding of the protein with various lipids and $A \beta$ [13-15]. Histologically, $A p o E$ and $A \beta$ can co-localise in the brain, and therefore their complementary roles have been studied extensively $[16,17]$. These studies have led to the association of the ApoE4 protein with lower $A \beta_{1-42}$ and higher tau levels observed in cerebrospinal fluid, increased brain atrophy and increased neocortical amyloid burden [7,18-20]. In contrast, ApoE2 is considered to be more cognitively protective than ApoE4; however, this is seemingly independent of actual $\mathrm{A} \beta$ pathology in the brain $[13,21]$.

Whilst the $A P O E$ gene is considered one of the strongest risk factors for late-onset $\mathrm{AD}$, the mechanisms and influence of actual plasma ApoE levels on the pathophysiology of $\mathrm{AD}$ remain unclear and require further elucidation. Therefore, we measured ApoE protein levels in plasma to further assess and determine the diagnostic value of $\mathrm{ApoE}$ as an $\mathrm{AD}$ blood biomarker. Given the prior knowledge of ApoE involvement in $A \beta$ metabolism mentioned above, we also evaluated the association of plasma ApoE on neocortical $A \beta$ burden as measured by positron emission tomography (PET).

Previously, we reported on the baseline ApoE data derived from the Australian Imaging, Biomarkers and Lifestyle Study of Ageing (AIBL) and showed a significant decrease in ApoE protein levels in the AD group compared with the cognitively 'normal' controls [22]. In the present study, we quantified ApoE data in the same subjects after 18 months of follow-up by utilising a more advanced enzyme-linked immunosorbent assay technology. This study not only validates our data in a time-dependent manner, within the same cohort, but also illustrates reproducibility with a different experimental method.

\section{Methods}

\section{The AIBL cohort}

The cohort recruitment process, including neuropsychological, lifestyle and mood assessments, have been described in detail previously [23]. In brief, in the AIBL study, researchers recruited a total of 1,166 participants over the age of 60 years at baseline, of whom 54 were excluded because of comorbid disorders or consent withdrawal. Using the National Institute of Neurological and Communicative Disorders and Stroke/Alzheimer's Disease and Related Disorders Association international criteria for AD diagnosis [24], a clinical review panel determined disease classifications at each assessment time point to ensure accurate and consistent diagnoses amongst the participants. According to these diagnostic criteria, participants were classified into one of three groups; $\mathrm{AD}$, mild cognitive impairment $(\mathrm{MCI})$ or healthy controls (HC). At baseline, there were a total of $768 \mathrm{HC}$, 133 subjects with $\mathrm{MCI}$ and 211 subjects with AD.

The AIBL study is a prospective, longitudinal study, following participants at 18-month intervals. In this report, we describe findings for 954 individuals who completed the full study assessment and corresponding blood sample collection at both baseline and 18-month follow-up. Of these 954 participants, 689 were classified as $\mathrm{HC}, 78$ as $\mathrm{MCI}$ and 187 as $\mathrm{AD}$.

The institutional ethics committees of Austin Health, St. Vincent's Health, Hollywood Private Hospital and Edith Cowan University granted ethical approval for the AIBL study. All volunteers gave their written informed consent prior to participating in the study.

\section{Sample collection and APOE genotyping}

Plasma was isolated from whole blood and collected in standard ethylenediaminetetraacetic acid tubes with prostaglandin $E_{1}(33.3 \mathrm{ng} / \mathrm{ml}$; Sapphire Biosciences, Waterloo, Australia) added. Upon completion of blood fractionation, samples were aliquoted and immediately stored in liquid nitrogen until required for analysis. 
DNA was isolated from whole blood using a QIAamp DNA Blood Midi Kit (Qiagen, Chadstone Centre, Australia) according to the manufacturer's protocol, and $A P O E$ genotype was determined through either PCR amplification and restriction enzyme digestions, as previously described [25], or through TaqMan genotyping assays (Life Technologies, Mulgrave, Australia) for rs7412 (Assay ID: C__904973_10) and rs429358 (Assay ID: C_3084793_20). For TaqMan assays, PCRs and real-time fluorescence measurements were carried out on a ViiA 7 real-time PCR system (Applied Biosystems, Mulgrave, Australia) using the TaqMan GTXpress Master Mix (Life Technologies) methodology per the manufacturer's instructions.

\section{Total apolipoprotein E assay}

Total plasma ApoE levels were measured using a commercial Luminex xMAP Human apolipoprotein kit (EMD Millipore, Billerica, MA, USA), a bead-based assay. This kit uses capture antibodies on the surface of fluorescently coated beads. Each microsphere is conjugated with a specific capture antibody-in this case, a specific human anti-ApoE antibody. Briefly, the plasma samples were thawed on ice, centrifuged for 10 minutes at $12,000 \times g$ and diluted 10,000 -fold using the supplied assay buffer diluents. Quality control and human ApoE calibrators were reconstituted in deionized water to give working solutions. Antibody-immobilised beads were prepared separately. The beads were vortexed for 1 minute and then incubated in a sonicating bath for 8 to 10 minutes. The beads were diluted using the provided diluents, and the final solution was sonicated and vortexed again just prior to loading onto the plate. Finally, all reagents were loaded onto the provided filter plate in the appropriate proportions as per the kit instructions, incubated for 1 hour and then vacuumdrained and washed. The detection antibodies and streptavidin-phycoerythrin were added for 30 minutes and vacuumed and washed in the same manner. Plates were read on the Bio-Plex 200 multiplexing instrument (Bio-Rad Laboratories, Gladesville, Australia). The assay sensitivity for ApoE was $0.10 \mathrm{ng} / \mathrm{ml}$, and the intra-assay and inter-assay precisions were $5 \%$ and $22 \%$, respectively.

\section{Brain imaging in a subset of the AIBL cohort}

A subset of the AIBL cohort $(n=287)$ underwent carbon11-labeled Pittsburgh Compound B positron emission tomography $\left({ }^{11} \mathrm{C}-\mathrm{PiB}-\mathrm{PET}\right)$ imaging at baseline to measure cerebral amyloid load as previously described [26]. PET standardised uptake value (SUV) data were summed and normalised to the cerebellar cortex SUV to form the region to cerebellar ratio (SUVR). Of the total 954 participants reported on here, 217 underwent PiB-PET imaging at 18-month follow-up.

\section{Statistical analysis}

Differences in demographics across clinical categories were assessed using one-way analysis of variance (ANOVA) for continuous data (age) and $\chi^{2}$ tests for categorised data (sex and $A P O E-\varepsilon 4$ carriage). Differences in ApoE levels between clinical classifications were assessed using ANOVA. Tukey's honestly significant difference (HSD) post hoc adjustment was applied to individual classification differences.

General linear models were used to assess correlations between SUVR and ApoE levels, and correlation coefficients $(\beta)$ and ApoE level specific $P$-values are reported. Receiver operating characteristic curves for predicting $\mathrm{PiB}$ status were calculated from predictions given by tenfold cross-validated random forest models, which have been shown previously [27] to have efficacy in creating blood-based predictors for PiB status. All statistical analyses were conducted using $\mathrm{R}$ software version 2.15.1 [28].

\section{Results}

Demographic data for the 18-month follow-up, including number of participants (both female and male) in each of the clinical categories, $A P O E-\varepsilon 4$ status, mean age and AIBL cohort are presented in Table 1. The average age was significantly higher in individuals with $\mathrm{MCI}$ (77.97 years) and $\mathrm{AD}$ (80.32 years) than in the $\mathrm{HC}$ category (73.51 years) $(P<0.001$, one-way ANOVA). The percentage of $A P O E-\varepsilon 4$-positive individuals was significantly higher in the $\mathrm{AD}(68.4 \%)$ and $\mathrm{MCI}(41 \%)$ groups than in the HC group (26.9\%). The number of female participants was generally higher than males in each of the clinical classification categories.

ApoE levels were significantly different across the diagnostic classification categories $(P=0.002)$ (Figure 1$)$. When controlling for age, sex and $A P O E-\varepsilon 4$ status, ApoE levels remained significantly different between clinical classifications $(\mathrm{p}<0.001)$. Post-hoc analysis (Tukey's HSD) revealed that lower ApoE levels were seen in individuals with $\mathrm{AD}(6.20 \mathrm{mg} / \mathrm{dl})$ when compared with $\mathrm{HC}(6.97 \mathrm{mg} / \mathrm{dl} ; \mathrm{p}=0.005)$. Total ApoE levels were significantly decreased in $A P O E$ - 44 carriers $(5.59 \mathrm{mg} / \mathrm{dl})$ compared with non-carriers $(7.41 \mathrm{mg} / \mathrm{dl}$; $\mathrm{p}<0.001$; Table 2) and remained so after correction for known pre-disposing factors, age and sex. This relationship also remained strong when the participants were stratified by clinical classification (with or without correction for age and sex) as well as stratification by sex (with or without correction for age); refer to Table 2 and Figure 2. Further, males had lower total ApoE levels $(6.12 \mathrm{mg} / \mathrm{dl})$ compared with females $(7.21 \mathrm{mg} / \mathrm{dl} ; \mathrm{p}=0.013)$ irrespective of clinical classification. ApoE levels were seen to have significant $(\mathrm{p}<0.05)$ differences across ApoE genotype classifications by one-way ANOVA with Tukey's 
Table 1 Demographic characteristics, including APOE- $\varepsilon 4$ frequency, of the study groups ${ }^{\mathrm{a}}$

\begin{tabular}{|c|c|c|c|c|}
\hline Categories & $\mathrm{HC}$ & $\mathrm{MCl}$ & $A D$ & $P$-value \\
\hline Count, $n$ & 689 & 78 & 187 & \\
\hline Age, yr & $73.51 \pm 6.78$ & $77.97 \pm 7.58$ & $80.32 \pm 7.79$ & $<0.001$ (one-way ANOVA) \\
\hline Sex, M/F & $285 / 404$ & $37 / 41$ & $76 / 111$ & 0.556 ( $x^{2}$ test $)$ \\
\hline APOE-ع4-positive, \% & 26.9 & 41 & 68.4 & $<0.001$ ( $x^{2}$ test $)$ \\
\hline
\end{tabular}

${ }^{\mathrm{a}} \mathrm{AD}$, Alzheimer's disease; APOE, Apolipoprotein $\mathrm{E} ; \mathrm{HC}$, Healthy controls; $\mathrm{MCl}$, Mild cognitive impairment. Values are mean \pm standard deviation or ratio (\%). Statistical analysis of age (in years) and sex of the participants was carried out using one-way analysis of variance (ANOVA), and APOE- $\varepsilon 4$ genotype frequency was performed using the $x^{2}$ test.

HSD post-hoc adjustment, refer to Figure 3 and Table 3. The only exceptions were between $\varepsilon 2 / \varepsilon 2$ and $\varepsilon 2 / \varepsilon 3 ; \varepsilon 3 / \varepsilon 4$ and $\varepsilon 4 / \varepsilon 4$ as well as comparisons with $\varepsilon 2 / \varepsilon 4$.

There was a significant association between ApoE and SUVR, with an increase in ApoE levels being associated with a decrease in SUVR $(\beta=-0.034, P=0.025)$ (Figure 4). Within different clinical categories, however, no correlation was observed between the two groups. The relationship was still evident when we corrected for age and sex in the model $(\beta=-0.040, P=0.009)$.

When ApoE levels were used to predict PiB-positive and PiB-negative status based on a cutoff of 1.5 SUVR [3], the area under the curve (AUC) was 59.83\% (95\% confidence interval (CI): $51.89 \%$ to $67.77 \%$ ), with a sensitivity of $57.68 \%$ (95\% CI: $51.76 \%$ to $63.60 \%$ ) and a specificity $56.76 \%$ (95\% CI: $50.61 \%$ to $62.91 \%$ ). When $A P O E$ genotype, age, sex and site were also added to the prediction model, the sensitivity and specificity were increased to $67.77 \%$ (95\% CI: $66.29 \%$ to $69.25 \%$ ) and $67.04 \%$ (95\% CI: $65.57 \%$ to $68.53 \%$ ), respectively, with an AUC of $79.12 \%$ (95\% CI: $78.14 \%$ to $80.10 \%)$. With regard to neocortical burden, a model for $A P O E$ genotype, age, sex and site alone obtained sensitivity of $69.74 \%$ (95\% CI: $63.27 \%$ to $76.21 \%$ ) and specificity of $67.76 \%$ (95\% CI: $62.34 \%$ to $73.18 \%$ ), respectively, with an AUC of $76.29 \%$ ( $95 \%$ CI: $69.54 \%$ to $83.05 \%$ ).

\section{Discussion}

In this study, we expanded the analysis of ApoE in a very well-characterised and well-described cohort, AIBL [23]. Plasma ApoE levels were measured at the 18-month time point. The multitude of data available for each of the AIBL participants allowed us to explore the relationship between ApoE protein levels and other related phenotypes to further reveal the pathways responsible for the onset of $\mathrm{AD}$. Whilst $A P O E$ genotype plays a significant role in determining the risk of an individual developing $\mathrm{AD}$, the role of $\mathrm{ApoE}$ at the protein level is not yet fully understood, and reported results have varied in the literature thus far.

The present study completely corroborates our previous findings that ApoE levels are significantly decreased in the MCI and AD groups compared with the HC [22]. These results remained significant even when we controlled

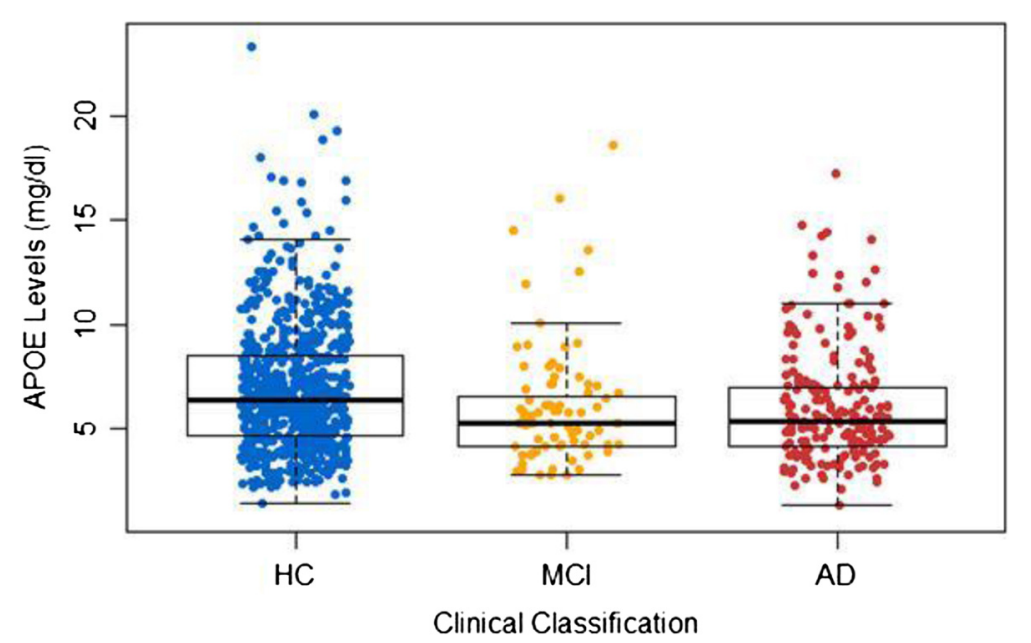

Figure 1 Apolipoprotein E protein levels across all clinical classifications in the AIBL cohort at 18 months. Data are presented as mean \pm standard deviation for absolute apolipoprotein E (ApoE) levels (mg/dl) across clinical classification categories of the Australian Imaging, Biomarkers and Lifestyle Study of Ageing (AIBL) cohort participants at the 18-month time point. One-way analysis of variance overall $P=0.002$, overall adjusted $P=0.001$ (adjusted for age, sex and APOE- $\varepsilon 4$ genotype), followed by post hoc Tukey's honestly significant difference test: healthy controls (HC) versus Alzheimer's disease (AD), $P=0.005 ; \mathrm{HC}$ versus mild cognitive impairment $(\mathrm{MCl}), P=0.997$; and $\mathrm{MCl}$ versus $\mathrm{AD}, P=0.064$. 
Table 2 Comparison of apolipoprotein E levels among different clinical classification and sex categories

\begin{tabular}{|c|c|c|c|c|c|}
\hline Categories & Total & Non- $\varepsilon 4$ carriers & $\varepsilon 4$ carriers & $P$ crude $^{a}$ & $P$ adj $^{\mathrm{a}}$ \\
\hline Total ApoE levels (mg/dl) & $6.75 \pm 3.00$ & $7.41 \pm 3.11$ & $5.59 \pm 2.40$ & $<0.001$ & $<0.001^{b}$ \\
\hline Total ApoE levels (HC) & $6.97 \pm 3.02$ & $7.45 \pm 3.10$ & $5.65 \pm 2.37$ & $<0.001$ & $<0.001^{\mathrm{b}}$ \\
\hline Total ApoE levels (MCl) & $6.17 \pm 3.02$ & $6.96 \pm 3.37$ & $5.02 \pm 1.97$ & 0.005 & $0.005^{\mathrm{b}}$ \\
\hline Total ApoE levels (AD) & $6.20 \pm 2.81$ & $7.39 \pm 3.05$ & $5.65 \pm 2.53$ & $<0.001$ & $<0.001^{\mathrm{b}}$ \\
\hline Total ApoE levels (female) & $7.21 \pm 3.10$ & $7.91 \pm 3.18$ & $5.94 \pm 2.87$ & $<0.001$ & $<0.001^{c}$ \\
\hline Total ApoE levels (male) & $6.12 \pm 2.74$ & $6.70 \pm 2.49$ & $5.12 \pm 2.18$ & $<0.001$ & $<0.001^{c}$ \\
\hline
\end{tabular}

$\mathrm{AD}$, Alzheimer's disease; $\mathrm{HC}$, Healthy controls; $\mathrm{MCl}$, Mild cognitive impairment, ${ }^{\mathrm{a}}$ Comparison between apolipoprotein $\mathrm{E}(\mathrm{APOE})-\varepsilon 4$ carriers and non- $\varepsilon 4$ carriers.

${ }^{\mathrm{b}} P$-values adjusted after controlling for age and sex. ${ }^{C} P$-values adjusted after controlling for age.

for age, sex and $A P O E-\varepsilon 4$ status. As the AIBL study is a longitudinal prospective study, these 18-month data validate the published baseline data for the same participants and are consistent with other studies [22,29-31]. Further, this report illustrates that ApoE levels are significantly decreased in $A P O E-\varepsilon 4$ carriers, even when stratified by clinical classification or sex. This was also consistent with our previously published data on baseline plasma ApoE [22]. These significant differences observed between the sexes are also supported by recent publications [31,32] in which authors have suggested that the $A P O E-\varepsilon 4$ link to $\mathrm{AD}$ is stronger in women. Those who are $A P O E-\varepsilon 4$ carriers have significantly lower ApoE central nervous system (CNS) and plasma ApoE levels, which may explain their propensity to develop $\mathrm{AD}[22,33,34]$. In this study, ApoE protein plasma levels are also defined by specific genotypes, with $\varepsilon 2 / \varepsilon 2$ participants having the highest ApoE levels and $\varepsilon 4 / \varepsilon 4$ participants having the lowest (Figure 3).
A decrease in the amount of available plasma ApoE among $\varepsilon 4$ allele carriers could have significant implications in the disease process. Given the involvement of ApoE in $\mathrm{A} \beta$ clearance and lipid transport, this seems mechanistically plausible. $A P O E-\varepsilon 4$ carriers have been shown to have increased amyloid burden, and this may be due to decreased clearance from the brain resulting from the limited ApoE available to bind $A \beta[17,19]$. Looking more closely at the literature regarding brain ApoE levels, this concept has also been demonstrated in targeted replacement mice, in which genotype clearly affected ApoE levels specifically in the CNS (with $\varepsilon 4 / \varepsilon 4$ mice having the least brain ApoE [34]). Also, reduced ApoE plasma and CNS levels correlated with the development of $\mathrm{AD}$, suggesting a direct consequence of having less ApoE. The ApoE4 isoform is reportedly less stable and may be preferentially degraded compared with ApoE3 in astrocytes, providing a possible biological explanation for the decrease in protein availability in this particular genotype [34].

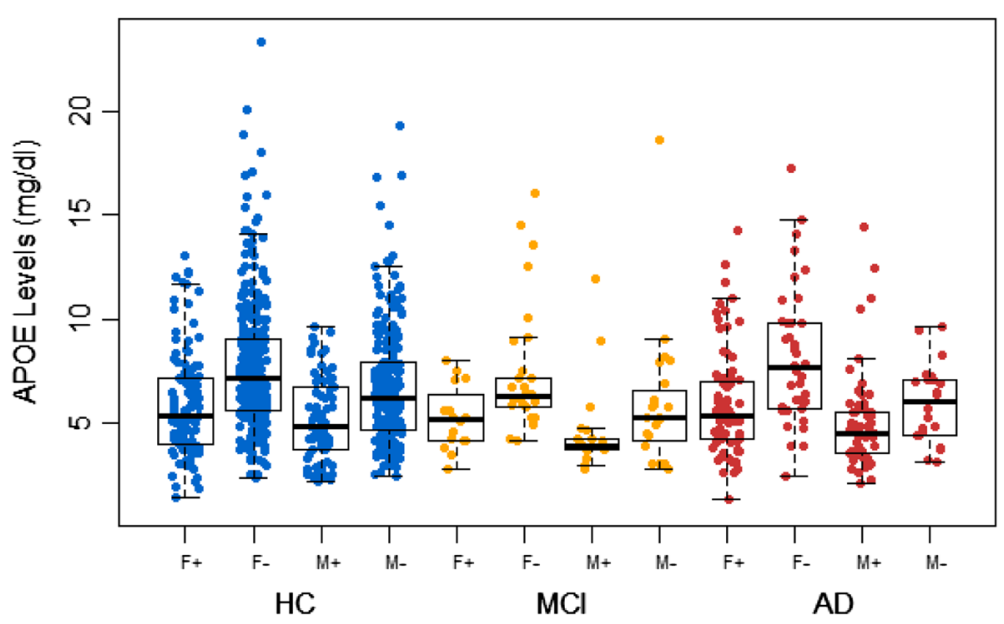

Figure 2 Absolute apolipoprotein E levels across clinical categories. Data are presented as means \pm standard deviations of apolipoprotein (ApoE) levels stratified by sex and APOE- $\varepsilon 4$ genotype in the Australian Imaging, Biomarkers and Lifestyle Study of Ageing (AIBL) cohort participants at the 18-month time point. One-way analysis of variance followed by post hoc Tukey's honestly significant difference test was carried out. Healthy controls ( $\mathrm{HC}$ ): females, $P<0.001$ for $\varepsilon 4(\mathrm{~F}+)$ versus non- $\varepsilon 4(\mathrm{~F}-$ ); males, $P<0.001$ for $\varepsilon 4(\mathrm{M}+$ ) versus non- $\varepsilon 4(\mathrm{M}-$ ). Mild cognitive impairment (MCI): females, $P=0.040$ for $\varepsilon 4$ versus non- $\varepsilon 4$; males, $P=0.539$ for $\varepsilon 4$ versus non- $\varepsilon 4$. Alzheimer's disease (AD): females, $P<0.001$ for $\varepsilon 4$ versus non- $\varepsilon 4$; males, $P=0.604$ for $\varepsilon 4$ versus non- $\varepsilon 4$. 


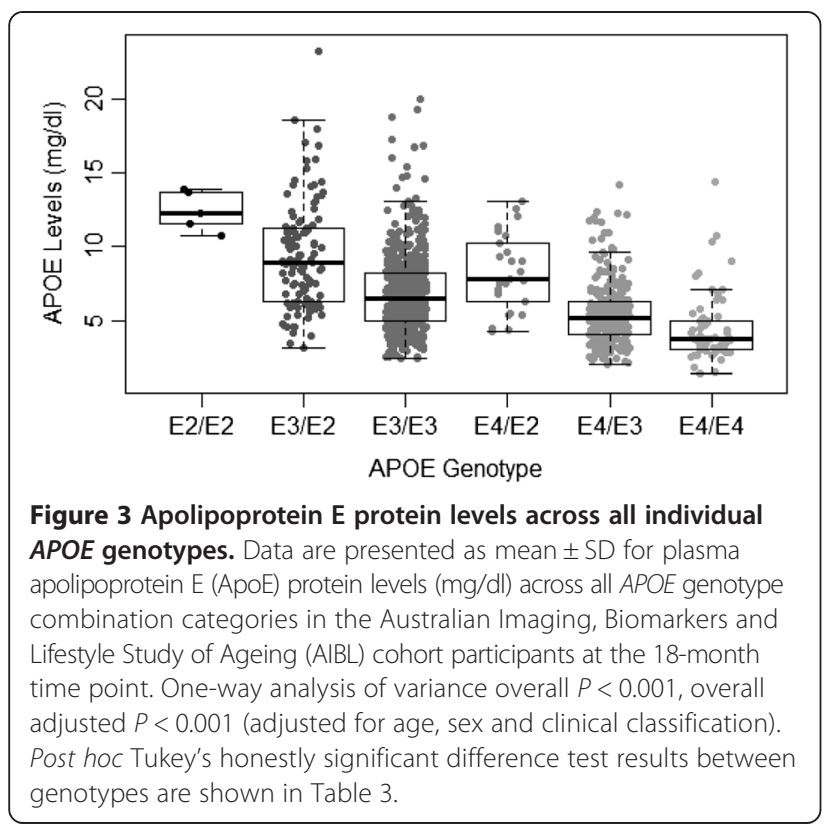

ApoE isoforms are known to differentially transport and regulate cholesterol levels because of their amino acid differences, with ApoE4 preferentially binding to low-density lipoprotein and ApoE2 or ApoE3 binding to high-density lipoprotein [34]. Cholesterol uptake is also in part dependent upon the ApoE isoform bound to the lipid because ApoE4-mediated cholesterol uptake has been shown to be lower [35,36]. It is likely, therefore, that peripheral ApoE levels, as determined by individual isoforms, have a direct effect on lipid transport and cholesterol levels. $A P O E-\varepsilon 4$ carriers, having insufficient ApoE, may have reduced distribution of cholesterol to neurons for important functions such as membrane maintenance, repair and synaptogenesis, which are crucial for learning and memory $[34,37,38]$. With AD subjects exhibiting lower ApoE levels in this cohort, this may have similar implications because over $68 \%$ of the AIBL AD group carries the APOE- $\varepsilon 4$ allele as well. Interestingly, researchers have used animal models to

Table 3 Comparison of apolipoprotein E levels among different APOE genotype categories

\begin{tabular}{llllll}
\hline & $\varepsilon 2 / \varepsilon 2$ & $\varepsilon 2 / \varepsilon 3$ & $\varepsilon 3 / \varepsilon 3$ & $\varepsilon 2 / \varepsilon 4$ & $\varepsilon 3 / \varepsilon 4$ \\
\hline Count, $n$ & 5 & 113 & 491 & 25 & 266 \\
$\varepsilon 2 / \varepsilon 3$ & 0.118 & & & & \\
$\varepsilon 3 / \varepsilon 3$ & $<0.001$ & $<0.001$ & & & \\
$\varepsilon 2 / \varepsilon 4$ & 0.020 & 0.474 & 0.156 & & \\
$\varepsilon 3 / \varepsilon 4$ & $<0.001$ & $<0.001$ & $<0.001$ & $<0.001$ & \\
$\varepsilon 4 / \varepsilon 4, N=55$ & $<0.001$ & $<0.001$ & $<0.001$ & $<0.001$ & 0.261 \\
\hline
\end{tabular}

$P$-values shown were calculated using one-way analysis of variance (ANOVA) followed by Tukey's honestly significant difference post hoc test. Refer to Figure 3.

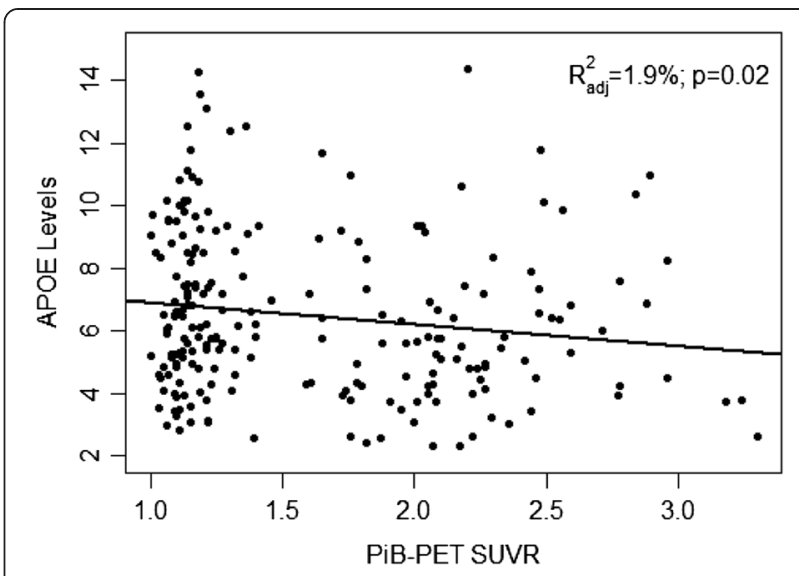

Figure 4 Correlation between total plasma apolipoprotein $\mathrm{E}$ levels (mg/dl) and cerebral amyloid burden as measured by carbon-11-labeled Pittsburgh Compound B positron emission tomography (PiB-PET). APOE, Apolipoprotein E; SUVR, Standardised uptake value ratio. $R_{\text {adj }}^{2}=1.9 \%, P=0.02$.

demonstrate that different ApoE isoforms also predict varying outcomes in response to CNS injury [13]. In this regard, ApoE deficient mice had a complete inability to recuperate from experimentally induced head injury, illustrating the essential role of this protein in neuronal repair [39].

Also of potential significance in considering the downstream effects of ApoE levels is that the cholinergic pathway is highly dependent upon lipid homeostasis for the synthesis of acetylcholine [40]. Cholinergic dysfunction is a well-documented feature of $\mathrm{AD}$, where many treatment strategies have revolved around augmenting levels of this particular neurotransmitter $[12,41]$. The link between these two systems is lipid maintenance, which illustrates the potential importance of ApoE in this pathway. Because the AD group in this study exhibited significantly lower levels of this protein, and considering the subsequent biological implications described here, plasma ApoE may be an important element in a predictive biomarker panel for early diagnosis.

The $A P O E-\varepsilon 4$ allele not only is considered a risk factor for $\mathrm{AD}$, but is now also being used as a predictor for cognitive decline. Cognitively normal $A P O E-\varepsilon 4$ allele carriers have been shown to exhibit an increase in amyloid burden as measured by PiB-PET [42]. Additionally, those who are cognitively healthy $A P O E-\varepsilon 4$ carriers have exhibited structural damage and associated cognitive decline compared with non-E4 carriers as observed by magnetic resonance imaging (MRI) [43,44]. With a clear reduction in ApoE protein levels associated with the carriage of the $A P O E-\varepsilon 4$ allele, and with the utilisation of MRI and PET neuroimaging, we are a step closer to understanding the consequences of reduced ApoE levels. 
In terms of the relationship between ApoE protein levels and amyloid burden in the present study, a subset of participants who underwent PiB-PET imaging were analysed separately with respect to plasma ApoE levels. A significant negative correlation was found between SUVR and ApoE levels, suggesting that lower circulating ApoE levels are associated with higher amyloid burden in the brain. Again, this supports our previous work, which demonstrated a similar significant result [22]. Given the relationship between ApoE isoforms and subsequent protein levels, ApoE could perhaps play an interchangeable role as a risk factor and/or biomarker.

To strengthen our findings, we used ApoE levels at the 18-month follow-up to predict neocortical $A \beta$ burden based on PiB-PET-determined SUVR. An AUC of $60 \%$ was observed for neocortical $A \beta$ burden predicted with ApoE protein levels alone; however, the addition of demographic and $A P O E$ genotype information to the model yielded an AUC of $80 \%$ (3\% above that of the demographic and $A P O E$ genotype information alone). The modest improvement in predicting neocortical $A \beta$ burden with the addition of plasma ApoE levels to the base model demonstrated a possible application for plasma ApoE levels in a clinical setting and its importance to the increased accuracy for potential population screening protocols to identify individuals at increased risk of developing AD.

\section{Conclusions}

The findings reported here are from the 18-month follow-up time point of the longitudinal AIBL study. The mean plasma ApoE levels are lower in the MCI and $\mathrm{AD}$ clinical categories than in the $\mathrm{HC}$ participants in the age, sex and $A P O E-\varepsilon 4$ genotype controlled data set. The differences in mean ApoE levels observed among the clinical categories are consistent with the previously published baseline results from AIBL and also reiterate that $A P O E-\varepsilon 4$ carriers have the lowest levels of plasma ApoE levels. This study gives the insight that lower levels of ApoE could have major implications in contributing to the progression of $\mathrm{AD}$ as also observed by its negative correlation with neocortical amyloid burden as measured by PiB-PET. On the basis of these consistent results derived from a large, well-characterised cohort, ApoE has the potential to become an important biomarker target for the early diagnosis of $\mathrm{AD}$.

\section{Abbreviations}

Aß: Amyloid- $\beta$; AD: Alzheimer's disease; AlBL: Australian Imaging, Biomarkers and Lifestyle Study of Ageing; ANOVA: Analysis of variance;

APOE: Apolipoprotein E; AUC: Area under the curve; Cl: Confidence interval;

CNS: Central nervous system; ${ }^{11} \mathrm{C}$-PiB-PET: Carbon-11-labeled Pittsburgh

Compound B positron emission tomography; HC: Healthy control;

HSD: Honestly significant difference; MCl: Mild cognitive impairment;
MRI: Magnetic resonance imaging; PET: Positron emission tomography; PiB: Pittsburgh Compound B; SD: Standard deviation; SUV: Standardised uptake value; SUVR: Standardised uptake value ratio.

\section{Competing interests}

Pfizer International has contributed financial support to assist with analysis of blood samples and to further the AIBL research program. SML has been a paid consultant for Alzhyme Pty Ltd. AIB is a shareholder in Prana

Biotechnology Ltd, Cogstate Ltd, Mesoblast Ltd, Brighton Biotech LLC and Eucalyptus Pty Ltd and is a paid consultant for Brighton Biotech LLC and Collaborative Medical Discovery LLC. CLM has interests in Prana Biotechnology and is an advisor to Eli Lilly. All the remaining authors declare that they have no competing interests.

\section{Authors' contributions}

VBG conceived of the study, and participated in its design, acquisition of data, analysis and interpretation of data and also helped to draft the manuscript. ACW participated in the design, acquisition, analysis and interpretation of data, carried out the immunoassays, and drafted the manuscript. SB performed all statistical analyses and helped to draft the manuscript. EH contributed to the interpretation of data and revised the manuscript critically for important intellectual content. SP contributed to the interpretation of data and revised the manuscript critically for important intellectual content. SML contributed data for analysis and revised the manuscript critically for important intellectual content. WLFL contributed to the interpretation of data and revised the manuscript critically for important intellectual content. AR contributed to the interpretation of data and revised the manuscript critically for important intellectual content. SR contributed to the interpretation of data and revised the manuscript critically for important intellectual content. DA contributed to the study design and intellectual input for the manuscript. LC contributed to the study design and intellectual input for the manuscript. SLM contributed to the study design and revised the manuscript critically for important content. CLM contributed to the study design and intellectual input for the manuscript. CCR contributed to the study design and intellectual input for the manuscript. AIB contributed to the study design and intellectual input for the manuscript. RNM conceived of the study design, interpretation of the data and revised the manuscript critically for important content. All authors read and approved the final manuscript.

\section{Authors' information}

AIBL Research Group: http://www.aibl.csiro.au/.

Cooperative Research Centre for Mental Health: http://www.mentalhealthcrc.com/.

\section{Acknowledgements}

Core funding for this study was provided by CSIRO, which was supplemented by "in-kind" contributions from study partners. This research is supported by the Science and Industry Endowment Fund (www.SIEF.org.au). The AIBL investigators thank Richard Head of CSIRO for initiating and facilitating the AIBL collaboration. The study also received support from the National Health and Medical Research Council (NHMRC) Australia via the Dementia Collaborative Research Centres (DCRC) program and through a project grant (APP1009292) awarded to RNM, SML and VBG. The authors also acknowledge the financial support of the Cooperative Research Centre (CRC) for Mental Health, an Australian Government Initiative. The McCusker Alzheimer's Research Foundation Inc contributed financial and in kind support to AIBL. We kindly thank all AIBL Research Group members (http:// aibl.csiro.au/about/aibl-research-team/) and those who took part as subjects in the study for their commitment and dedication to helping advance research into the early detection and causation of $A D$.

\section{Author details}

${ }^{1}$ Centre of Excellence in Alzheimer's Disease Research and Care, School of Medical Sciences, Edith Cowan University, 270 Joondalup Drive, Joondalup 6027, Australia. ${ }^{2}$ McCusker Alzheimer's Research Foundation, Hollywood Medical Centre, 85 Monash Avenue, Suite 22, Nedlands 6009, Australia. ${ }^{3} \mathrm{CSIRO}$ Computational Informatics, Preventative Health Flagship, 65 Brockway Road, Floreat 6014, Australia. ${ }^{4}$ Cooperative Research Centre for Mental Health, Carlton, VIC 3053, Australia. ${ }^{5}$ Florey Institute of Neuroscience and Mental Health, Parkville, VIC 3052, Australia. ${ }^{6}$ Academic Unit for Psychiatry of Old Age, Department of Psychiatry, The University of Melbourne, St Vincent's Aged Psychiatry Service, St George's Hospital, Melbourne, VIC 3065, Australia. 
${ }^{7}$ National Ageing Research Institute, Parkville, VIC 3052, Australia. ${ }^{8} \mathrm{CSIRO}$ Preventative Health Flagship, Adelaide, SA 5000, Australia. ${ }^{9} \mathrm{CSIRO}$, Parkville, VIC 3052, Australia. ${ }^{10}$ Mental Health Research Institute, The University of Melbourne, Parkville, VIC 3052, Australia. ${ }^{11}$ Centre for Neuroscience, The University of Melbourne, Parkville, VIC 3010, Australia. ${ }^{12}$ Department of Nuclear Medicine \& Centre for PET, Austin Health, Heidelberg, VIC 3084, Australia. ${ }^{13}$ School of Psychiatry and Clinical Neurosciences, The University of Western Australia, Nedlands 6009, Australia.

\section{Received: 10 June 2014 Accepted: 6 February 2015}

\section{Published online: 20 February 2015}

\section{References}

1. Tarawneh R, Holtzman DM. Biomarkers in translational research of Alzheimer's disease. Neuropharmacology. 2010;59:310-22.

2. Thambisetty M, Lovestone S. Blood-based biomarkers of Alzheimer's disease: challenging but feasible. Biomark Med. 2010;4:65-79.

3. Rowe CC, Ellis KA, Rimajova M, Bourgeat P, Pike KE, Jones $G$, et al. Amyloid imaging results from the Australian Imaging, Biomarkers and Lifestyle (AIBL) study of aging. Neurobiol Aging. 2010;31:1275-83.

4. Rowe CC, Ng S, Ackermann U, Gong SJ, Pike K, Savage G, et al. Imaging $\beta$-amyloid burden in aging and dementia. Neurology. 2007;68:1718-25.

5. Villemagne $V L$, Burnham S, Bourgeat $P$, Brown B, Ellis KA, Salvado O, et al. Amyloid $\beta$ deposition, neurodegeneration, and cognitive decline in sporadic Alzheimer's disease: a prospective cohort study. Lancet Neurol. 2013;12:357-67.

6. Saunders AM, Strittmatter WJ, Schmechel D, St George-Hyslop PH, Pericak-Vance MA, Joo SH, et al. Association of apolipoprotein E allele $\varepsilon 4$ with late-onset familial and sporadic Alzheimer's disease. Neurology. 1993:43:1467-72

7. Polvikoski T, Sulkava R, Haltia M, Kainulainen K, Vuorio A, Verkkoniemi A, et al. Apolipoprotein $E$, dementia, and cortical deposition of $\beta$-amyloid protein. N Engl J Med. 1995;333:1242-7.

8. Corder EH, Saunders AM, Strittmatter WJ, Schmechel DE, Gaskell PC, Small $\mathrm{GW}$, et al. Gene dose of apolipoprotein E type 4 allele and the risk of Alzheimer's disease in late onset families. Science. 1993;261:921-3.

9. Rebeck GW, Reiter JS, Strickland DK, Hyman BT. Apolipoprotein E in sporadic Alzheimer's disease: allelic variation and receptor interactions. Neuron. 1993;11:575-80.

10. Mahley RW. Apolipoprotein E: cholesterol transport protein with expanding role in cell biology. Science. 1988;240:622-30.

11. Boyles JK, Zoellner CD, Anderson L, Kosik LM, Pitas RE, Weisgraber KH, et al. A role for apolipoprotein E, apolipoprotein A-I, and low density lipoprotein receptors in cholesterol transport during regeneration and remyelination of the rat sciatic nerve. J Clin Invest. 1989;83:1015-31.

12. Poirier J. Apolipoprotein E, and Alzheimer's disease: a role in amyloid catabolism. Ann N Y Acad Sci. 2000;924:81-90.

13. Liu CC, Kanekiyo T, Xu H, Bu G. Apolipoprotein E and Alzheimer disease: risk, mechanisms and therapy. Nat Rev Neurol. 2013:9:106-18.

14. LaDu MJ, Falduto MT, Manelli AM, Reardon CA, Getz GS, Frail DE. Isoform-specific binding of apolipoprotein E to $\beta$-amyloid. J Biol Chem. 1994;269:23403-6.

15. Yang DS, Smith JD, Zhou Z, Gandy SE, Martins RN. Characterization of the binding of amyloid- $\beta$ peptide to cell culture-derived native apolipoprotein E2, E3, and E4 isoforms and to isoforms from human plasma. J Neurochem. 1997;68:721-5

16. Strittmatter WJ, Saunders AM, Schmechel D, Pericak-Vance M, Enghild J, Salvesen GS, et al. Apolipoprotein E: high-avidity binding to $\beta$-amyloid and increased frequency of type 4 allele in late-onset familial Alzheimer disease. Proc Natl Acad Sci U S A. 1993:90:1977-81.

17. Strittmatter WJ, Weisgraber KH, Huang DY, Dong LM, Salvesen GS, Pericak-Vance $M$, et al. Binding of human apolipoprotein $E$ to synthetic amyloid $\beta$ peptide: isoform-specific effects and implications for late-onset Alzheimer disease. Proc Natl Acad Sci U S A. 1993;90:8098-102.

18. Evans KC, Berger EP, Cho CG, Weisgraber KH, Lansbury Jr PT. Apolipoprotein $E$ is a kinetic but not a thermodynamic inhibitor of amyloid formation: implications for the pathogenesis and treatment of Alzheimer disease. Proc Natl Acad Sci U S A. 1995;92:763-7.
19. Schmechel DE, Saunders AM, Strittmatter WJ, Crain BJ, Hulette CM, Joo SH, et al. Increased amyloid $\beta$-peptide deposition in cerebral cortex as a consequence of apolipoprotein E genotype in late-onset Alzheimer disease. Proc Natl Acad Sci U S A. 1993:90:9649-53.

20. Vemuri P, Wiste HJ, Weigand SD, Knopman DS, Shaw LM, Trojanowski JQ, et al. Effect of apolipoprotein E on biomarkers of amyloid load and neuronal pathology in Alzheimer disease. Ann Neurol. 2010;67:308-16.

21. Berlau DJ, Corrada MM, Head E, Kawas CH. APOE $\varepsilon 2$ is associated with intact cognition but increased Alzheimer pathology in the oldest old. Neurology. 2009;72:829-34.

22. Gupta VB, Laws SM, Villemagne VL, Ames D, Bush Al, Ellis KA, et al. Plasma apolipoprotein $\mathrm{E}$ and Alzheimer disease risk: the AIBL study of aging. Neurology. 2011;76:1091-8.

23. Ellis KA, Bush Al, Darby D, De Fazio D, Foster J, Hudson P, et al. The Australian Imaging, Biomarkers and Lifestyle (AIBL) study of aging: methodology and baseline characteristics of 1112 individuals recruited for a longitudinal study of Alzheimer's disease. Int Psychogeriatr. 2009;21:672-87

24. Tierney MC, Fisher RH, Lewis AJ, Zorzitto ML, Snow WG, Reid DW, et al. The NINCDS-ADRDA Work Group criteria for the clinical diagnosis of probable Alzheimer's disease: a clinicopathologic study of 57 cases. Neurology. 1988:38:359-64.

25. Hixson JE, Vernier DT. Restriction isotyping of human apolipoprotein E by gene amplification and cleavage with Hhal. J Lipid Res. 1990;31:545-8.

26. Pike KE, Savage G, Villemagne VL, Ng S, Moss SA, Maruff $P$, et al. $\beta$-amyloid imaging and memory in non-demented individuals: evidence for preclinical Alzheimer's disease. Brain. 2007;130:2837-44.

27. Burnham SC, Faux NG, Wilson W, Laws SM, Ames D, Bedo J, et al. A blood-based predictor for neocortical $A \beta$ burden in Alzheimer's disease: results from the AIBL study. Mol Psychiatry. 2014;19:519-26.

28. R: A language and environment for statistical computing. http://www.R-project.org/. Accessed 14 Mar 2015.

29. Siest $G$, Bertrand $P$, Qin B, Herbeth B, Serot JM, Masana L, et al. Apolipoprotein E polymorphism and serum concentration in Alzheimer's disease in nine European centres: the ApoEurope study. Clin Chem Lab Med. 2000;38:721-30.

30. Wang C, Yu JT, Wang HF, Jiang T, Tan CC, Meng XF, et al. Meta-analysis of peripheral blood apolipoprotein E levels in Alzheimer's disease. PLoS One. 2014;9:e89041

31. Cruchaga C, Kauwe JS, Nowotny P, Bales K, Pickering EH, Mayo K, et al. Cerebrospinal fluid APOE levels: an endophenotype for genetic studies for Alzheimer's disease. Hum Mol Genet. 2012;21:4558-71.

32. Altmann A, Tian L, Henderson WW, Greicius MD. Alzheimer's Disease Neuroimaging Initiative Investigators. Sex modifies the APOE-related risk of developing Alzheimer disease. Ann Neurol. 2014;75:563-73.

33. Raffai RL, Dong LM, Farese Jr RV, Weisgraber KH. Introduction of human apolipoprotein E4 "domain interaction" into mouse apolipoprotein E. Proc Natl Acad Sci U S A. 2001;98:11587-91.

34. Riddell DR, Zhou H, Atchison K, Warwick HK, Atkinson PJ, Jefferson J, et al. Impact of apolipoprotein $E$ (ApoE) polymorphism on brain ApoE levels. J Neurosci. 2008;28:11445-53.

35. Rapp A, Gmeiner B, Hüttinger M. Implication of apoE isoforms in cholesterol metabolism by primary rat hippocampal neurons and astrocytes. Biochimie. 2006:88:473-83.

36. Leoni $\mathrm{V}$. The effect of apolipoprotein $\mathrm{E}(\mathrm{ApoE})$ genotype on biomarkers of amyloidogenesis, tau pathology and neurodegeneration in Alzheimer's disease. Clin Chem Lab Med. 2011:49:375-83.

37. Pfrieger FW. Role of cholesterol in synapse formation and function. Biochim Biophys Acta. 2003;1610:271-80

38. Mauch DH, Nägler K, Schumacher S, Göritz C, Müller EC, Otto A, et al. CNS synaptogenesis promoted by glia-derived cholesterol. Science. 2001;294:1354-7.

39. Chen Y, Lomnitski L, Michaelson DM, Shohami E. Motor and cognitive deficits in apolipoprotein E-deficient mice after closed head injury. Neuroscience. 1997:80:1255-62.

40. Blusztajn JK, Liscovitch M, Richardson UI. Synthesis of acetylcholine from choline derived from phosphatidylcholine in a human neuronal cell line. Proc Natl Acad Sci U S A. 1987;84:5474-7.

41. Perry EK. The cholinergic system in old age and Alzheimer's disease. Age Ageing. 1980;9:1-8. 
42. Pike KE, Ellis KA, Villemagne VL, Good N, Chetelat G, Ames $\mathrm{D}$, et al. Cognition and $\beta$-amyloid in preclinical Alzheimer's disease: data from the AIBL study. Neuropsychologia. 2011;49:2384-90.

43. Espeseth T, Westlye LT, Fjell AM, Walhovd KB, Rootwelt H, Reinvang I. Accelerated age-related cortical thinning in healthy carriers of apolipoprotein $\mathrm{E}$ ع4. Neurobiol Aging. 2008;29:329-40.

44. Fennema-Notestine C, Panizzon MS, Thompson WR, Chen CH, Eyler LT, Fischl B, et al. Presence of ApoE $\varepsilon 4$ allele associated with thinner frontal cortex in middle age. J Alzheimers Dis. 2011;26:49-60.

\section{Submit your next manuscript to BioMed Central} and take full advantage of:

- Convenient online submission

- Thorough peer review

- No space constraints or color figure charges

- Immediate publication on acceptance

- Inclusion in PubMed, CAS, Scopus and Google Scholar

- Research which is freely available for redistribution 